\title{
O DESENVOLVIMENTO MOTOR E A AQUISIÇÃO DE HABILIDADES MOTORAS EM AUTISTAS
}

\author{
Rossana de Vasconcelos Pugliese Vito ${ }^{12 *}$ \& Darlan Santos ${ }^{1}$
}

VITO, R.V.P. \& SANTOS, D. O desenvolvimento motor e a aquisição de habilidades Motoras em autistas. Perspectivas Online: Biológicas \& Saúde, v.10, n.34, p.1-15, 2020.

\section{RESUMO}

O desenvolvimento motor e as habilidades motoras em crianças autistas ainda permanecem pouco compreendidas. Estudos sugerem fortes relações entre o transtorno do espectro autista (TEA) com déficits do desenvolvimento motor e a aquisição de habilidades motoras, mas apesar disso, ainda hoje, essa questão não é considerada como critério diagnóstico. Fator intrigante entre os pesquisadores da área. A presente pesquisa realizou uma Revisão Integrativa da Literatura (RIL), com o objetivo de relacionar estudos sobre o desenvolvimento motor e a aquisição das habilidades motoras em crianças com TEA quando comparadas com crianças típicas. A pesquisa foi relizada na base de dados do Pub Med, através dos descritores "autismo" e "desenvolvimento motor" e "habilidades motoras", após a aplicação dos critérios de inclusão e exclusão foram selecionados quinze artigos para análise. Os estudos incluídos apresentam um consenso razoável sobre a existência de atrasos motores em crianças com TEA, contudo em sua quase unanimidade, apontam para a necessidade de pesquisas mais aprofundadas por motivos diversos, seja pelo baixo número de participantes ou pelas dificuldades de isolar as caracterísitcas autísticas, em seus diferentes níveis.

Palavras-chave: Autismo; Desenvolvimento Motor; Habilidades Motoras.

\footnotetext{
${ }^{1}$ Unisaude Educacional - UNISAUDE - Laboratório de Estudos da Criança - LABESCRI - Estrada Rodrigues Alves, 299/ Loja E, Taquara, Rio de Janeiro, RJ, CEP: 22713-372, Brasil;

${ }^{2}$ Universidade Estácio de Sá - UNESA - Av. Dom Helder Câmara, 5474; $4^{\circ}$ piso Norte Shopping, Cachambi, RJ, CEP: 20771-004, Brasil.

(*) e-mail: rossanapugli@gmail.com
} 


\title{
MOTOR DEVELOPMENT AND MOTOR SKILL ACQUISITION IN AUTIST INDIVIDUALS
}

\author{
Rossana de Vasconcelos Pugliese Vito ${ }^{1,2 *}$ \& Darlan Santos ${ }^{1}$
}

VITO, R.V.P. \& SANTOS, D. Motor development and motor skill acquisition in autist individuals. Perspectivas Online: Biológicas \& Saúde, v.10, n.34, p.1-15, 2020.

\begin{abstract}
Motor development and motor skills in autistic children are still poorly understood. The studies suggest strong relationships between autism spectrum disorder (ASD) with deficits in motor development and acquisition of motor skills, but despite this, even today, this issue is not considered as a test method. Intriguing factor among researchers in the area. This research carried out an Integrative Literature Review (RIL), with the objective of relating studies on motor development and the acquisition of motor skills in children with ASD, when

research was carried out at Pub Med database, using the descriptors "autism" and "motor development" and "motor skills", after applying the inclusion and exclusion requirements of the items selected for analysis. The studies included a reasonable consensus on the presence of engine delays in children with ASD, however, their almost unanimity, pointed to a need for more in-depth research for different reasons, whether the number of participants was smaller or the difficulties of isolating as characteristics automatic, at its different levels.
\end{abstract} compared with typical children. The

Keywords: Autism; Motor Development; Motor Skills.

\footnotetext{
${ }^{1}$ Unisaude Educacional - UNISAUDE - Laboratório de Estudos da Criança - LABESCRI - Estrada Rodrigues Alves, 299/ Loja E, Taquara, Rio de Janeiro, RJ, CEP: 22713-372, Brasil;

${ }^{2}$ Universidade Estácio de Sá - UNESA - Av. Dom Helder Câmara, 5474; $4^{\circ}$ piso Norte Shopping, Cachambi, RJ, CEP: 20771-004, Brasil.

(*) e-mail: rossanapugli@gmail.com
} 


\section{INTRODUÇÃO}

O diagnóstico do transtorno do espectro autista (TEA) tem aumentado de forma exponencial na população mundial, chegando numa proporção de um caso a cada cento e sessenta crianças (Organização Mundial da Saúde, 2017), este número difere em diversos países e em países subdesenvolvidos não se têm um controle exato da prevalência, caso do Brasil.

O autismo é um transtorno neurobiológico multifatorial do desenvolvimento, que compromete as áreas de interação e linguagem, apresentando um quadro sintomático muito extenso, envolvendo os aspectos emocionais, cognitivos, motores e sensoriais (ASSUMPÇÃO JÚNIOR; KUCZYNSKI, 2015; RELVAS, 2015). Sua etiologia ainda é muito difícil de ser precisa, o que a literatura aponta hoje é para uma causa biológica, envolvendo um grande componente genético e diversos fatores ambientais (ASSUMPÇÃO JÚNIOR; KUCZYNSKI, 2015; RELVAS, 2015; MERCADANTES; LECKMAN, 2013).

De acordo com a Associação Americana de Psiquiatria (APA, 2013), através do lançamento da quinta edição do Manual Diagnóstico e Estatístico de Transtornos Mentais (DSM-V), houveram mudanças na forma da caracterizar o TEA e também nos seus critérios de diagnósticos. O DSM-V é utilizado para facilitar o estabelecimento de diagnósticos mais confiáveis nos transtornos mentais, trazendo uma classificação desses transtornos a seus critérios associados, tudo associado e em harmonização com a Classificação Internacional de Doenças (CID), elaborado pela Organização Mundial da Saúde (OMS). Tendo sua utilização realizada por diversos profissionais e pesquisadores das áreas mais distintas, dentre elas: médicos, psicólogos, terapeutas, biólogos, dentre outros (APA, 2013).

O Manual afirma que o TEA é um transtorno que afeta o neurodesenvolvimento e se caracteriza por se manifestar desde cedo no desenvolvimento infantil, geralmente antes dos três anos de idade, e por trazer prejuízos no funcionamento social, pessoal, acadêmico e posteriormente profissional (APA, 2013). O DSM-V alerta que devido aos sintomas do TEA variarem de acordo com a fase de desenvolvimento da criança, alguns desses sintomas podem ser mascarados por mecanismo compensatórios, mas os critérios diagnósticos podem ser enquadrados com base em informações retrospectivas, contudo o comportamento atual apresentado pela criança deve trazer prejuízo nas áreas que caracterizam o TEA (APA, 2013). Segundo o DSM-V as características essenciais para o diagnóstico do TEA são: padrões restritos e repetitivos de comportamentos e interesses em atividades, prejuízos persistentes na comunicação social e na interação social. Esses critérios diagnósticos devem estar presentes no início da infância, limitando ou prejudicando de alguma forma o funcionamento diário do indivíduo.

A tabela a seguir relaciona os critérios de diagnóstico estabelecidos pelo DSM-V. É essencial ressaltar que o TEA, a partir dessa publicação, passa a ser descrito em níveis de gravidade, indo do leve- 1 (onde é exigido pouco apoio), moderado-2 (apoio substancial) e severo-3. 
Tabela 1- Critérios diagnósticos TEA - DSM-V. Fonte: adaptado do American Psychiatric Association (2013).

\begin{tabular}{|c|c|}
\hline $\mathrm{A}$ & $\begin{array}{l}\text { Déficits persistentes na comunicação social e na interação social em múltiplos contextos, conforme } \\
\text { manifestado pelo que segue, atualmente ou por história prévia (os exemplos são apenas ilustrativos, e } \\
\text { não exaustivos): }\end{array}$ \\
\hline A1 & $\begin{array}{l}\text { Déficits na reciprocidade socioemocional, variando, por exemplo, de abordagem social anormal e } \\
\text { dificuldade para estabelecer uma conversa normal a compartilhamento reduzido de interesses, } \\
\text { emoções ou afeto, a dificuldade para iniciar ou responder a interações sociais }\end{array}$ \\
\hline A2 & $\begin{array}{l}\text { Déficits nos comportamentos comunicativos não verbais usados para interação social, variando, por } \\
\text { exemplo, de comunicação verbal e não verbal pouco integrada a anormalidade no contato visual e } \\
\text { linguagem corporal ou déficits na compreensão e uso gestos, a ausência total de expressões faciais e } \\
\text { comunicação não verbal. }\end{array}$ \\
\hline A3 & $\begin{array}{l}\text { Déficits para desenvolver, manter e compreender relacionamentos, variando, por exemplo, de } \\
\text { dificuldade em ajustar o comportamento para se adequar a contextos sociais diversos a dificuldade em } \\
\text { compartilhar brincadeiras imaginativas ou em fazer amigos, a ausência de interesse por pares. }\end{array}$ \\
\hline B & $\begin{array}{l}\text { Padrões restritos e repetitivos de comportamento, interesses ou atividades, conforme manifestado por } \\
\text { pelo menos dois dos seguintes, atualmente ou por história prévia (os exemplos são apenas } \\
\text { ilustrativos, e não exaustivos) }\end{array}$ \\
\hline B1 & $\begin{array}{l}\text { Movimentos motores, uso de objetos ou fala estereotipados ou repetitivos (p. ex., estereotipias } \\
\text { motoras simples, alinhar brinquedos ou girar objetos, ecolalia, frases idiossincráticas). }\end{array}$ \\
\hline B2 & $\begin{array}{l}\text { Insistência nas mesmas coisas, adesão inflexível a rotinas ou padrões ritualizados de comportamento } \\
\text { verbal ou não verbal (p. ex., sofrimento extremo em relação a pequenas mudanças, dificuldades com } \\
\text { transições, padrões rígidos de pensamento, rituais de saudação, necessidade de fazer o mesmo } \\
\text { caminho ou ingerir os mesmos alimentos diariamente). }\end{array}$ \\
\hline B3 & $\begin{array}{l}\text { Interesses fixos e altamente restritos que são anormais em intensidade ou foco (p. ex., forte apego a } \\
\text { ou preocupação com objetos incomuns, interesses excessivamente circunscritos ou perseverativos). }\end{array}$ \\
\hline B4 & $\begin{array}{l}\text { Hiper ou hiporreatividade a estímulos sensoriais ou interesse incomum por aspectos sensoriais do } \\
\text { ambiente (p. ex., indiferença aparente a dor/temperatura, reação contrária a sons ou texturas } \\
\text { específicas, cheirar ou tocar objetos de forma excessiva, fascinação visual por luzes ou movimento). }\end{array}$ \\
\hline $\mathrm{C}$ & $\begin{array}{l}\text { Os sintomas devem estar presentes precocemente no período do desenvolvimento (mas podem não se } \\
\text { tornar plenamente manifestos até que as demandas sociais excedam as capacidades limitadas ou } \\
\text { podem ser mascarados por estratégias aprendidas mais tarde na vida). }\end{array}$ \\
\hline $\mathrm{D}$ & $\begin{array}{l}\text { Os sintomas causam prejuízo clinicamente significativo no funcionamento social, profissional ou em } \\
\text { outras áreas importantes da vida do indivíduo no presente }\end{array}$ \\
\hline $\mathrm{E}$ & $\begin{array}{l}\text { Essas perturbações não são mais bem explicadas por deficiência intelectual (transtorno do } \\
\text { desenvolvimento intelectual) ou por atraso global do desenvolvimento. Deficiência intelectual ou } \\
\text { transtorno do espectro autista costumam ser comórbidos; para fazer o diagnóstico da Comorbidade de } \\
\text { transtorno do espectro autista e deficiência intelectual, a comunicação social deve estar abaixo do } \\
\text { esperado para o nível geral do desenvolvimento. }\end{array}$ \\
\hline
\end{tabular}

Muitos estudos examinaram aspectos sociais, emocionais e comportamentais em crianças com TEA e existe um grande corpo de trabalho focado em intervenções e fatores contextuais e ambientais. Mas a sintomatologia motora ainda é pouco compreendida e não está incluída nos critérios de diagnóstico do transtorno do espectro (TEA), apesar de alguns estudos sugerirem a presença desses distúrbios (PAQUET et al., 2015).

O desenvolvimento motor e a aquisição de habilidades motoras de crianças autistas encontram-se na literatura em diversos relatos clínicos sobre as funções motoras e seus déficits (WILSON et al., 2018). A dificuldade nas habilidades motoras em indivíduos com TEA pode vir a causar impactos na vida cotidiana e social, pois estas habilidades se encontram presentes em todos os contextos da vida diária. E a existência de disfunções motoras nos primeiros meses de vida, combinados com problemas sociais e sinais de comunicação posteriores, podem ser um indicador precoce no diagnóstico. No entanto, a triagem precoce é difícil em termos metodológicos devido às diferentes formas clínicas 
associadas (LANDA et al., 2012).

Alguns estudos apontam características motoras observadas em idade precoce de crianças diagnosticadas com TEA, são elas: hipotonia (KANNER, 1943; ADRIEN et al., 1993; MAESTRO et al., 2005), estereotipias motoras (LOH et al., 2007; WATT et al., 2008) e instabilidade postural (IVERSON \& WOZNIAK, 2007; ORNITZ et al., 1977).

Apesar disso PAQUET et al. (2016) ressaltam que a imputabilidade de distúrbios motores ainda é pouco clara, pois é difícil atribuir sintomas motores precoces ao TEA, ou a atrasos no desenvolvimento, ou ainda, em comorbidade com outras disfunções, haja vista que dificuldades motoras são comuns em distúrbios do desenvolvimento neurológico. Relatam ainda que embora a existência de deficiências motoras não seja um preditivo da sintomatologia autista, ela pode constituir um fator agravante no prognóstico do TEA, o que torna essencial levar em consideração a sondagem neuromotora e motora na triagem precoce, a fim de estabelecer rapidamente a direção do tratamento.

Favoráveis a esse pensamento, ASSUMPÇÃO JÚNIOR (2015) e KUCZYNSKI (2015) reforçam ainda que, o diagnóstico precoce gera um prognóstico mais favorável para os indivíduos com TEA, a intervenção sobre as funções motoras também deve ser realizada de forma precoce, objetivando um aumento nas funções motoras, que irão ajudar nas atividades da vida cotidiana bem como na vida social dessas crianças.

Diante do dilema da existência ou não de evidencias claras sobre distúrbios motores em crianças com TEA, o objetivo da presente pesquisa é relacionar os estudos com a temática do desenvolvimento motor e a aquisição de habilidades motoras em autistas, através de uma Revisão Integrativa da Literatura (RIL).

Para composição desse estudo foram relacionadas algumas das maiores pesquisas na área, publicadas nos últimos cinco anos na Pub Med, trazendo concordâncias e discordâncias entre os autores e provocando a comunidade científica a continuar pensando sobre o assunto

\section{METODOLOGIA}

Com o propósito de revisar métodos, teorias e estudos empíricos sobre o tópico em questão, através de resumo e análise, foi adotada nesse estudo a Revisão Integrativa. Para WHITEMORE e KNAFL (2005), o "termo integrativa tem origem na integração de opiniões, conceitos ou ideias provenientes das pesquisas utilizadas no método".

Assim realizou-se a pesquisa exploratória, qualitativa e descritiva, com procedimentos metodológicos da Revisão Integrativa da Literatura (RIL), operacionalizados através de procedimentos de busca eletrônica, conduzidos em nível nacional e internacional. Para tanto foi acessada a base de dados Pub Med. Foram utilizados os descritores de saúde Mesh: "autismo" $e$ "desenvolvimento motor" e "habilidades motoras". Esses descritores foram utilizados isoladamente ou combinados entre si, com o auxílio dos operadores booleanos $A N D$ e $O R$, no período de março de 2015 a março de 2020.

Para formular a pergunta do estudo foi utilizada a estratégia conhecida como PICOT (2015). O anagrama PICOT: População, Intervenção/exposição, comparação, desfechos/outcome e tipo de estudo. P: crianças; I: TEA; C: Crianças Típicas; 
O: habilidades motoras e desenvolvimento motor; T: ensaios clínicos, estudos transversais e estudos de corte.

A pergunta formulada que norteia a presente pesquisa é: como se dá o desenvolvimento motor e das habilidades motoras em crianças com TEA quando comparadas com crianças típicas?

O trabalho de pesquisa seguiu as mesmas fases propostas em pesquisas de revisão, quais sejam: a) explicitação do problema a ser investigado; b) formulação da pergunta norteadora da pesquisa; c) rastreamento nas bases de dados disponíveis; d) coleta e análise crítica dos resultados das pesquisas incluídas; e) discussão dos resultados encontrados e f) apresentação de quadro sintético dos resultados da Revisão Integrativa da Literatura.

Como procedimentos foram adotados alguns critérios de inclusão e de exclusão nas buscas de artigos, os chamados critérios de elegibilidade. As RIL recuperadas foram analisadas em relação aos critérios de inclusão. Os títulos e resumos dos artigos foram analisados, sendo considerados passíveis de inclusão, na Revisão Integrativa e o artigo que teve como resposta afirmativa em todas as 4 (quatro) questões:

a) O conteúdo do artigo aborda o processo de desenvolvimento motor e a aquisição de habilidades motoras em crianças autistas?

b) A abordagem do artigo está voltada especificamente para o natural processo de desenvolvimento motor em crianças autistas e suas possíveis implicações, sem mencionar possíveis intervenções?

c) O conteúdo do artigo se refere a comportamentos motores alterados de forma global?

d) A abordagem é intrínseca a área da saúde?

Foram excluídos da pesquisa os estudos que obtiveram resposta negativa, em qualquer uma das quatro questões formuladas, não correspondendo, portanto, ao conteúdo de interesse da pergunta inicial.

Após cada artigo ser submetido a uma avaliação dos títulos e resumos, foi realizada a leitura integral, eliminados quando não se enquadravam nos critérios de busca previamente determinados. Na sequencia os dados foram extraídos, sob a perspectiva qualitativa e expostos no formato de relatório descritivo, conforme tabela. Para o registro dos dados foram elaborados quadros contendo as seguintes informações: autor, título do artigo, ano, faixa etária do estudo, revista publicado, $\mathrm{N}$ da pesquisa, abordagem e país. 


\section{RESULTADOS}

Como resultado da análise foram encontrados:

\section{Títulos obtidos do \\ banco de dados \\ $\mathrm{n}=136$}

Títulos retidos

$\mathrm{n}=57$

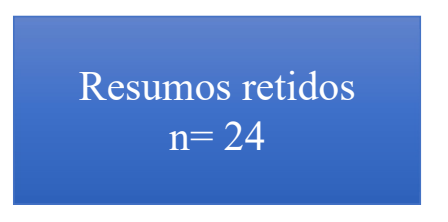

Artigos para

avaliação $n=24$

\section{Duplicados $n=0$}<smiles>CCCCCCCC</smiles>

Excluídos pelo titulo $n=79$<smiles>C1CC2CC12</smiles>

Excluídos pelo resumo $n=33$<smiles>C1C2CC1C2</smiles>

Excluídos pelo texto na integra $n=7 \longrightarrow$

Excluídos por não terem o texto na íntegra $=2$

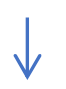

\section{Estudos incluídos}

$\mathrm{n}=15$ 


\begin{tabular}{|c|c|c|c|c|c|c|}
\hline $\begin{array}{l}\text { PUSPONEGORO, H. et } \\
\text { al. Gross Motor Profile } \\
\text { and Its Association with } \\
\text { Socialization Skills in } \\
\text { Children with Autism } \\
\text { Spectrum Disorders }\end{array}$ & 2016 & $\begin{array}{l}18 \\
\text { meses - } \\
6 \text { anos }\end{array}$ & $\begin{array}{l}\text { Pediatrics and } \\
\text { Neonatology }\end{array}$ & 40 & $\begin{array}{l}\text { Comparar a } \\
\text { habilidade motora } \\
\text { grossa de crianças } \\
\text { com TEA com } \\
\text { crianças em } \\
\text { desenvolvimento } \\
\text { típico } \\
\end{array}$ & $\begin{array}{l}\text { Indonés } \\
\text { ia }\end{array}$ \\
\hline $\begin{array}{l}\text { IZADI-NAJAFABADI, } \\
\text { S. et al. Implicit and } \\
\text { explicit motor learning: } \\
\text { Application to children } \\
\text { with Autism Spectrum } \\
\text { Disorder (ASD) }\end{array}$ & 2015 & $\begin{array}{l}7-11 \\
\text { anos }\end{array}$ & $\begin{array}{l}\text { Research in } \\
\text { Developmental } \\
\text { Disabilities }\end{array}$ & 30 & $\begin{array}{l}\text { Aprendizagem } \\
\text { motora implícita e } \\
\text { explícita, da mesma } \\
\text { tarefa,em crianças } \\
\text { com TEA } \\
\text { comparadas com } \\
\text { crianças em } \\
\text { desenvolvimento } \\
\text { típico }\end{array}$ & USA \\
\hline $\begin{array}{l}\text { TRAVERS, B. et al. } \\
\text { Longitudinal } \\
\text { development of manual } \\
\text { motor ability in autism } \\
\text { spectrum disorder from } \\
\text { childhood to mid- } \\
\text { adulthood relates to } \\
\text { adaptive daily living } \\
\text { skills }\end{array}$ & 2017 & $\begin{array}{l}5-40 \\
\text { anos }\end{array}$ & $\begin{array}{l}\text { Developmental } \\
\text { Science }\end{array}$ & 90 & $\begin{array}{l}\text { Estudo longitudinal } \\
\text { da } \\
\text { força de preensão em } \\
\text { indivíduos com TEA } \\
(\mathrm{n}=90) \text { em } \\
\text { comparação com } \\
\text { indivíduos típicos ( } \mathrm{n} \\
=56) \text {, com idades } \\
\text { entre } 5 \text { e } 40 \text { anos. o } \\
\text { objetivo deste estudo } \\
\text { é examinar a } \\
\text { trajetória de } \\
\text { desenvolvimento da } \\
\text { habilidade motora no } \\
\text { TEA desde a infância } \\
\text { até a idade adulta. }\end{array}$ & USA \\
\hline $\begin{array}{l}\text { HELLENDOORN, A. et } \\
\text { al. Motor functioning, } \\
\text { exploration, visuospatial } \\
\text { cognition and language } \\
\text { development in } \\
\text { preschool children with } \\
\text { autism }\end{array}$ & 2015 & $\begin{array}{l}14-15 \\
\text { meses }\end{array}$ & $\begin{array}{l}\text { Research in } \\
\text { Developmental } \\
\text { Disabilities }\end{array}$ & $\begin{array}{l}549 \\
\text { crianç } \\
\text { as } \\
\text { com } \\
\text { TEA } \\
\text { e } \\
\text { crianç } \\
\text { as } \\
\text { com } \\
\text { outro } \\
\text { s } \\
\text { atraso } \\
\text { s / } \\
\text { distúr } \\
\text { bios } \\
\text { no } \\
\text { desen } \\
\text { volvi } \\
\text { ment } \\
\text { o. }\end{array}$ & $\begin{array}{l}\text { Este longitudinal } \\
\text { entre a motricidade } \\
\text { fina precoce, a } \\
\text { cognição } \\
\text { visuoespacial, } \\
\text { exploração e } \\
\text { desenvolvimento da } \\
\text { linguagem na pré- } \\
\text { escola } \\
\text { crianças com TEA e } \\
\text { crianças com outros } \\
\text { atrasos / distúrbios no } \\
\text { desenvolvimento. }\end{array}$ & $\begin{array}{l}\text { Holand } \\
\text { a }\end{array}$ \\
\hline $\begin{array}{l}\text { CHINELLO, A., et al. } \\
\text { Persistent primary } \\
\text { reflexes affect motor } \\
\text { acts: Potential } \\
\text { implications for autism } \\
\text { spectrum disorder. }\end{array}$ & 2018 & $\begin{array}{l}12-17 \\
\text { meses }\end{array}$ & $\begin{array}{l}\text { Research in } \\
\text { Developmental } \\
\text { Disabilities }\end{array}$ & 34 & $\begin{array}{l}\text { O estudo sugere que a } \\
\text { persistência de } \\
\text { reflexos primitivos } \\
\text { pode alterar a } \\
\text { trajetória do } \\
\text { desenvolvimento } \\
\text { Motor de crianças } \\
\text { com TEA. }\end{array}$ & $\begin{array}{l}\text { ITÁLI } \\
\text { A }\end{array}$ \\
\hline
\end{tabular}




\begin{tabular}{|c|c|c|c|c|c|c|}
\hline $\begin{array}{l}\text { STEVENSON et al. } \\
\text { Retrospectively Assessed } \\
\text { Early Motor and Current } \\
\text { Pragmatic Language } \\
\text { Skills in Autistic and } \\
\text { Neurotypical Children }\end{array}$ & 2017 & $\begin{array}{l}-18 \\
\text { anos }\end{array}$ & $\begin{array}{l}\text { Perceptual and } \\
\text { Motor Skills }\end{array}$ & $\begin{array}{l}76 \\
\text { cuida } \\
\text { dores } \\
\text { de } 31 \\
\text { crianç } \\
\text { as } \\
\text { neuro } \\
\text { típica } \\
\text { s e } 45 \\
\text { crianç } \\
\text { as } \\
\text { autist } \\
\text { as }\end{array}$ & $\begin{array}{l}\text { O estudo relata que as } \\
\text { primeiras } \\
\text { atipicalidades no } \\
\text { espectro do autismo } \\
\text { estão no } \\
\text { domínio motor, não } \\
\text { na comunicação } \\
\text { social. Apoiando a } \\
\text { ideia de que desafios } \\
\text { ou atrasos motores } \\
\text { podem desempenhar } \\
\text { um papel na } \\
\text { comunicação autista, } \\
\text { destacando o valor de } \\
\text { explorar ainda mais o } \\
\text { papel da dispraxia no } \\
\text { autismo } \\
\text { comunicação. Ou } \\
\text { seja, o atraso na } \\
\text { comunicação pode } \\
\text { ser oriundo do atraso } \\
\text { motor, haja vista as } \\
\text { conexões neurais } \\
\text { entre ambos. }\end{array}$ & USA \\
\hline $\begin{array}{l}\text { MOSCONI MW et al. } \\
\text { Sensorimotor } \\
\text { dysfunctions as primary } \\
\text { features of autism } \\
\text { spectrum disorders }\end{array}$ & 2015 & & $\begin{array}{l}\text { SCIENCE } \\
\text { CHINA Life } \\
\text { Sciences }\end{array}$ & & $\begin{array}{l}\text { Revisão de Literatura } \\
\text { com o objetivo de } \\
\text { enfatizar a disfunção } \\
\text { motora no TEA e } \\
\text { evidência de } \\
\text { alterações nos } \\
\text { sistemas cerebrais } \\
\text { subjacentes }\end{array}$ & USA \\
\hline $\begin{array}{l}\text { KETCHESON et al. The } \\
\text { effects of an early motor } \\
\text { skill intervention on } \\
\text { motor skills, levels of } \\
\text { physical activity, and } \\
\text { socialization in young } \\
\text { children with autism } \\
\text { spectrum disorder: A } \\
\text { pilot study }\end{array}$ & 2016 & $\begin{array}{l}4-6 \\
\text { anos }\end{array}$ & Autism & 11 & $\begin{array}{l}\text { Estudo piloto sobre } \\
\text { os efeitos da } \\
\text { estimulação motora } \\
\text { precoce no } \\
\text { desenvolvimento de } \\
\text { crianças com TEA }\end{array}$ & USA \\
\hline $\begin{array}{l}\text { IVERSON, M. J. et al. } \\
\text { Early motor abilities in } \\
\text { infants at heightened vs. } \\
\text { low risk for ASD: A } \\
\text { Baby Siblings Research } \\
\text { Consortium (BSRC) } \\
\text { study }\end{array}$ & 2019 & $\begin{array}{l}6-36 \\
\text { meses }\end{array}$ & $\begin{array}{l}\text { J. Abnorm } \\
\text { Psychol }\end{array}$ & 648 & $\begin{array}{l}\text { Desenvolvimento } \\
\text { motor anormal e risco } \\
\text { de diagnostico de } \\
\text { TEA }\end{array}$ & EUA \\
\hline $\begin{array}{l}\text { LeBARTON, E. S.; } \\
\text { LANDA, R. J. Infant } \\
\text { motor skill predicts later } \\
\text { expressive language and } \\
\text { autism spectrum disorder } \\
\text { diagnosis }\end{array}$ & 2019 & $\begin{array}{l}6-36 \\
\text { meses }\end{array}$ & $\begin{array}{l}\text { J. Infant } \\
\text { Behavior and } \\
\text { Development }\end{array}$ & 140 & $\begin{array}{l}\text { Desenvolvimento } \\
\text { motor anormal e risco } \\
\text { de diagnostico de } \\
\text { TEA }\end{array}$ & $\overline{\text { EUA }}$ \\
\hline $\begin{array}{l}\text { SRINIVASAN, S.M.; } \\
\text { BHAT, A.N. Differences } \\
\text { in means end exploration } \\
\text { between infants at risk }\end{array}$ & 2019 & $\begin{array}{l}9-24 \\
\text { meses }\end{array}$ & $\begin{array}{l}\text { Developmental } \\
\text { Psychobiology }\end{array}$ & 23 & $\begin{array}{l}\text { Verificar habilidades } \\
\text { motoras e resolução } \\
\text { de problemas em } \\
\text { bebes com alto risco }\end{array}$ & EUA \\
\hline
\end{tabular}




\begin{tabular}{|c|c|c|c|c|c|c|}
\hline $\begin{array}{l}\text { for autism and typically } \\
\text { developing infants in the } \\
\text { first } 15 \text { months of life }\end{array}$ & & & & & $\begin{array}{l}\text { de diagnostico de } \\
\text { TEA comparados } \\
\text { com bebes típicos }\end{array}$ & \\
\hline $\begin{array}{l}\text { MANICOLO, O. et al. } \\
\text { Gait in children with } \\
\text { infantile/atypical autism: } \\
\text { Age-dependent decrease } \\
\text { in gait variability and } \\
\text { associations with motor } \\
\text { skills }\end{array}$ & 2019 & $\begin{array}{l}4-17 \\
\text { anos }\end{array}$ & $\begin{array}{l}\text { European } \\
\text { Journal of } \\
\text { Paediatric } \\
\text { Neurology }\end{array}$ & 68 & $\begin{array}{l}\text { Avaliação da marcha } \\
\text { e associação desta } \\
\text { com marcos motores, } \\
\text { habilidades motoras e } \\
\text { idade em crianças } \\
\text { com TEA } \\
\text { comparados com } \\
\text { crianças típicas }\end{array}$ & Suiça \\
\hline $\begin{array}{l}\text { PAQUET, A. Et al. } \\
\text { Nature of motor } \\
\text { impairments in autism } \\
\text { spectrum disorder: A } \\
\text { comparison with } \\
\text { developmental } \\
\text { coordination disorder }\end{array}$ & 2019 & $\begin{array}{l}4-15 \\
\text { anos }\end{array}$ & $\begin{array}{l}\text { Journal of } \\
\text { Clinical and } \\
\text { Experimental } \\
\text { Neuropsycholog } \\
\text { y }\end{array}$ & 76 & $\begin{array}{l}\text { Comparar a natureza } \\
\text { das deficieincias } \\
\text { motoras em crianças } \\
\text { com TEA com } \\
\text { crianças que } \\
\text { apresentem } \\
\text { Distúrbios de } \\
\text { coordenação sem } \\
\text { TEA }\end{array}$ & França \\
\hline
\end{tabular}

\section{DISCUSSÃO}

Os estudos incluídos nessa revisão integrativa da literatura apresentam um consenso razoável sobre a existência de atrasos motores, em crianças com TEA, contudo essa sintomatologia também pode ser encontrada para outras deficiências do desenvolvimento, o que ainda inviabiliza a afirmação. Algumas relações entre os estudos foram encontradas, principalmente no que tange as interações sociais, o desenvolvimento da linguagem e a importância de incluir a programação motora como parte dos serviços de intervenção precoce oferecidos as crianças com desordem do espectro autista (KETCHESON et al., 2016).

Harris (2017) afirma que durante o primeiro ano de vida, o pediatra não deve descartar a possibilidade de TEA em bebês com os comportamentos motores como hipotonia, apraxia e déficits no controle postural. Investigações mais profundas (por exemplo, avaliação audiológica, investigações genéticas) devem contribuir para fornecer um diagnóstico completo e minucioso de TEA. Os pediatras devem considerar a avaliação do desenvolvimento motor, bem como habilidades sociais e de comunicação, durante o acompanhamento rotineiro de crianças com TEA.

Já correlacionando as habilidades motoras precoces com habilidades pragmáticas posteriores, crianças autistas exibiram menos habilidades motoras do que crianças neurotípicas. De fato, habilidades motoras foram um melhor preditor de um diagnóstico do espectro do autismo do que pontuações em uma medida da linguagem pragmática atual. Esses resultados destacam o papel importante das habilidades motoras nos distúrbios do espectro do autismo e seu diagnóstico (STEVENSON et al., 2017).

Já CHINELLO et al. (2018) narram que estudos anteriores já relataram que assimetrias posturais persistentes estavam presentes apenas em $40 \%$ das crianças com TEA. A variabilidade desses déficits em todo o espectro é um desafio que provavelmente reflete o quadro clínico e heterogeneidade etiológica do TEA. Ao mesmo tempo, constitui uma oportunidade única para identificar subtipos de doenças. A partir dessas considerações, iniciaram um estudo com uma população geral de crianças de 12 a 17 meses. O estudo buscou 
entender se havia alguma relação entre a persistência de reflexos primitivos que envolvem o uso da mão e da boca e (1) idade do bebê, (2) repertório motor de bebês e (3) características autísticas subclínicas medidas em seus pais. Os resultados revelaram uma maior inibição dos reflexos primitivos em crianças mais velhas do que em crianças mais jovens. Esse resultado sustenta uma explicação maturacional das inibições dos reflexos primitivos: à medida que o sistema nervoso se desenvolve, os reflexos primitivos são inibidos ou transformados. Os resultados revelaram também a presença de reflexos primitivos além do período normal de tempo (12 meses), confirmando trabalhos anteriores. Os resultados revelaram que a persistência dos reflexos primitivos pelos participantes está relacionada a traços autistas subclínicos em seus parentes próximos: pais com traços autísticos subclínicos mais altos têm bebês com uma persistência mais forte de reflexos do que pais com características autísticas subclínicas mais baixas. No total, os resultados deste estudo estão alinhados com todos aqueles estudos que sugeriram observar o domínio motor como um sinal precoce promissor de TEA.

No decorrer do desenvolvimento infantil, há sinais que devem ser investigados. Em um estudo com crianças de 14 e 15 meses, Hellendoorn et al. (2015) narraram que a coordenação motora fina pode estar diretamente relacionada com desenvolvimento da linguagem, porque as habilidades motoras finas facilitam a interação entre físico e o social, em especial por possibilitar o desenvolvimento expressivo da linguagem. Se a coordenação motora fina, por algum motivo, não se desenvolver dentro do marco esperado, poderia influenciar no desenvolvimento da linguagem? Não seria esse um importante marcador, na busca de respostas sobre o atraso motor e a criança com TEA, haja vista que o atraso na linguagem é uma característica já comprovada?

Contudo LeBarton e Landra (2019) afirmam que as dificuldades motoras podem ser um indicador de risco precoce do TEA e podem interferir em habilidades subsequentes de linguagem expressiva. E enfatizam algo importante, que se ignoradas questões relacionadas ao desenvolvimento motor em crianças com TEA, pode haver um efeito em cascata e a interrupção precoce do mesmo.

Dos 18 meses a seis anos, as crianças com TEA apresentam coordenação motora global abaixo da média, em relação a crianças típicas. Vinte por cento das crianças com TEA têm deficiências motoras graves, com diminuição de habilidades que requerem coordenação complexa. E há uma tendência a menores habilidades sociais em crianças com TEA que tenham deficiências motoras graves (Pusponegoro et al., 2016).

Travers et al. (2017) relatam mais um aspecto intrigante em seu estudo, o grupo com TEA demonstrou desenvolvimento motor atípico caracterizado por similar desempenho durante a infância, mas desempenho cada vez pior da adolescência até a idade adulta. Seus resultados de testes de força de preensão sugerem que indivíduos com TEA podem experimentar dificuldades motoras maiores na adolescência e na idade adulta, enfatiza ainda que, o desempenho motor manual no TEA está relacionado às habilidades adaptativas da vida diária.

Dentre os estudos incluídos na revisão, até aqui foram relacionadas as investigações que sugerem fortes relações entre o transtorno do espectro autista (TEA) com déficits do desenvolvimento motor e a aquisição de habilidades motoras. Contudo hoje, ainda não é possível afirmar com segurança que as habilidades motoras devem ser inseridas na sintomatologia do TEA. Isso se deve ao fato da ausência de comprovação científica necessária, com significância estatística. A seguir serão apresentados os estudos encontrados

Persp. online: biol. \& saúde, Campos dos Goytacazes, 34 (10) 1-15, 2020

https://ojs3.perspectivasonline.com.br 
que exibiram hipótese nula, mas que também apresentam grande contribuição para a dialeticidade científica.

Manicolo et al. (2019) relata que crianças com TEA apresentaram maior variabilidade da marcha do que as crianças dos grupos controle, indicando sim um padrão de caminhada menos regular, mas essa questão não pode ser associada a marcos motores antes da caminhada. A maior variabilidade da marcha em crianças com TEA mostraram uma diminuição dependente da idade, sugerindo que a regularidade da marcha converge para a de crianças em desenvolvimento típico.

Srinivasan e Bhat (2019) citam que não houve diferenças entre os grupos bebês típicos e bebês com risco para autismo, para testes de exploração de meios e habilidades na resolução de problemas. Ambos os grupos demonstraram melhorias estratificadas, relacionadas à idade nas habilidades motoras e cognitivas.

Iverson et al. (2019) narram que, em suas pesquisas com bebês, nenhuma evidência foi encontrada de sinais motores específicos que aparecem precocemente associados a um diagnóstico posterior de TEA.

Nesse sentido, a presente revisão de literatura vem reforçar que a imputabilidade de distúrbios motores ainda é pouco clara para atribuir sintomas motores precoces ao TEA. Mas reforça que é essencial levar em consideração a sondagem neuromotora e motora na triagem precoce, a fim de estabelecer rapidamente a direção do tratamento.

\section{CONCLUSÕES}

As pesquisas recentes sobre habilidades motoras e desenvolvimento motor em crianças com TEA destacam que há um desenvolvimento anormal por parte dessas crianças, porém, ainda não se pode considerar estas características como preditoras do TEA e, consequentemente, sua gravidade. Analisado o prognóstico no contexto mais amplo, os déficits motores funcionam como um fator agravante e nesse sentido, é de fundamental importância considerar os déficits motores e suas divergências com o intuito de realizar uma intervenção precoce e também dar um direcionamento específico para o tratamento. A dificuldade no desenvolvimento da linguagem e no desenvolvimento de habilidades sociais foram alguns fatores encontrados que se agravam em função dos déficits motores.

Novas pesquisas na área devem ser realizadas, principalmente na direção de utilizar os déficits motores como critérios diagnósticos para o TEA.

\section{REFERÊNCIAS}

ADRIEN, J. L., LENOIR, P., MARTINEAU, J., PERROT, A., HAMEURY, L., LARMANDE, C., \& SAUVAGE, D. Blind ratings of early symptoms of autism based upon family home movies. Journal of the American Academy of Child \& Adolescent Psychiatry, 32(3), 617-626, 1993.

ALANIZ, M.L.; GALIT, E.; NECESITO, C.I.; \& ROSARIO, E.R. Hand Strength, Handwriting, and Functional Skills in Children With Autism. Am J Occup Ther. Jul-Aug;69 (4).2015. 
AMERICAN PSYCHIATRIC ASSOCIATION. Diagnostic and Statistical Manual of Mental Disorders: Diagnostic and Statistical Manual of Mental Disorders, Fifth Edition. Arlington, VA: American Psychiatric Association, 2013.

ASSUMPÇÃO JÚNIOR, F. B.; \& KUCZYNSKI, F. Autismo infantil: novas tendências e perspectivas. 2 ed. Atheneu, 2015.

CHINELLO, A.; DI GANGI, V.; \& VALENZA, E. Persistent primary reflexes affect motor acts: Potential implications for autism spectrum disorder, Research in Developmental Disabilities. V.83: 287-295, 2018.

FAIGENBAUM, A.D.; WESTCOTT, W.L.; LOUD, R.L.; \& LONG, C. The Effects of Different Resistance Training Protocols on Muscular Strength and Endurance Development in Children. Pediatrics. Jul;104(1):e5. 1999.

FAIGENBAUM, A.D.; LLOYD, R.S.; MACDONALD, J.; \& MYER, G.D. Citius, altius, fortius: beneficial effects of resistance training for young athletes. British Journal of Sports Medicine. Jan;50(1):3-7. 2016.

HARRIS, S.R. Early motor delays as diagnostic clues in autism spectrum disorder. European journal of pediatrics, v. 176, n. 9, p. 1259-1262, 2017.

HELLENDOORN, A.; WIJNROKS, L.; VAN DAALEN, E.; DIETZ, C.; BUITELAAR, J.K.; \& LESEMAN, P. Motor functioning, exploration, visuospatial cognition and language development in preschool children with autism. Research in Developmental Disabilities. V.39: 32-42, 2015.

IVERSON, J. M., \& WOZNIAK, R. H. Variation in vocal-motor development in infant siblings of children with autism. Journal of Autism \& Developmental Disorders, 37(1), 158-170, 2007.

IVERSON, J. M.; SHIC, F.; WALL, C.A.; CHAWARSKA,K.; CURTIN, S.; ESTES, A.; GARDNER, J.M.; HUTMAN, T.; LANDA, R.J.; LEVIN, A.R.; LIBERTUS, K.; MESSINGER, D.S.; NELSON, C.A.; OZONOFF, S.; SACREY, L-A.R.; SHEPERD, K.; STONE, W.L.; TAGER-FLUSBERG, H.B.; WOLFF, J.J.; ...YOUNG, G.S. Early motor abilities in infants at heightened vs. low risk for ASD: A Baby Siblings Research Consortium (BSRC) study. J Abnorm Psychol. V.128 (1): 69-80, 2019.

IZADI-NAJAFABADI, S.; MIRZAKHANI-ARAGHI, N.; MIRI-LAVASANI, N.; NEJATI, V.; \& PASHAZADEH-AZARI, Z. Implicit and explicit motor learning: Application to children with Autism Spectrum Disorder (ASD). Research in developmental disabilities, v. 47, p. 284-296, 2015.

KANNER, L. Autistic disturbances of affective contact. Nervous Child, 2, 217-250, 1943.

KETCHESON, L.; HAUCK, J.; \& ULRICH, D. The effects of an early motor skill intervention on motor skills, levels of physical activity, and socialization in young children with autism spectrum disorder: a pilot study. Autism, V. 21 issue: 4, page(s): 481-492. 2016.

LANDA, R. J., GROSS, A. L., STUART, E. A., \& BAUMAN, M. Latent class analysis of early developmental trajectory in baby siblings of children with autism. Journal of Child Psychology and Psychiatry, 53(9), 986-996, 2012. 
Le BARTON, E. S.; \& LANDA, R. J. Infant motor skill predicts later expressive language and autism spectrum disorder diagnosis. Infant Behavior and Development. V.54: 37-47, 2019.

LESINSKI, M. PRIESKE, O.; \& GRANACHER, U. EFFECTS and dose-response relationships of resistance training on Physical performance in youth athletes: a systematic review and meta-analysis. J. Sports Med; 50(13): 781-95, 2016.

LOH, A.; SOMAN, T.; BRIAN, J.; BRYSON, S. E.; ROBERTS, W.; SZATMARI, P.; SMITH, I.M.; \& ZWAIGENBAUM, L. Stereotyped motor behaviors associated with autism in high-risk infants: A pilot videotape analysis of a sibling sample. Journal of Autism and Developmental Disorders, 37(1), 25-36, 2007.

LLOYD, R.S.; FAIGENBAUM, A.D.; STONE, M.H.; OLIVER, J.L.; JEFFREYS, I.; MOODY, J.A.; BREWER, C.; PIERCE, K.C.; MACCAMBRIDGE, T.M.; HOWARD, R.; HERRINGTON, L.; HAINLINE, B.; MICHELI, L.J.; JAQUES, R.; KRAEMER, W.J.; MCBRIDE, M.G.; BEST, T.M.; CHU, D.A.; ALVAR, B.A.; \& MYER, G.D. Position statement on youth resistance training: the 2014 international consensus. Br. J. Sports Med; 48:498-505. 2014.

LLOYD, R.S.; CRONIN, J.B.; FAIGENBAUM, A.D.; et al. National Strength and Conditioning Association position statement on long-term athletic development. J. Strength Cond. Res. 30:1491-509. 2016.

MAESTRO, S., MURATORI, F., CESARI, A., CAVAllARO, M. C., PAZIENTE, A., PECINI, C., ... SOMMARIO, C. Course of autism signs in the first year of life. Psychopathology, 38(1), 26-31, 2005.

MANICOLO, O.; BROTZMANN, M.; ARX, P. H.; GROB, A.; WEBER,P. Gat in children with infantile/atypical autism: age-dependent decrease in gait variability and associations with motors skills. European Journal of Paediatric Neurology. V. 23, issue 1, p 117-125, 2019.

MERCADANTE, M.T.; LECKMAN, J.F. More than two dozen "autisms". Rev. Bras. Psiquiatric. Vol.35, n.1. São Paulo. 2013.

MOSCONI, M.W. Feedforward and feedback motor control abnormalities implicate cerebellar dysfunctions in autism spectrum disorder. J Neurosci. Feb 4;35(5):2015-25. 2015.

MOSCONI, M.W.; SWEENEY, J. A. Sensorimotor dysfunctions as primary features of autism spectrum disorders. Sci China Life Sci. V. 58(10):1016-23, 2015

ORGANIZAÇÃO MUNDIAL DA SAÚDE, Autism Spectrum Disorders. 2017. Disponível em: https://www.who.int/news-room/fact-sheets/detail/autism-spectrum-disorders acesso em: abril 2020.

ORNITZ, E. M., GUTHRIE, D., \& FARLEY, A. H. The early development of autistic children. Journal of Autism and Childhood Schizophrenia, 7(3), 207-229, 1977.

PAQUET, A. et al. Current knowledge on motor disorders in children with autism spectrum disorder (ASD). Child neuropsychology, v. 22, n. 7, p. 763-794, 2016. 
PAQUET, A.; OLLIAC, B.; GOLSE, B.; VAIVRE-DOURET, L. Nature of motor impairments in autism spectrum disorder: A comparison with developmental coordination disorder. Journal of Clinical and Experimental Neuropsychology. V. 41(1):1-14, 2019.

PEÑAHERRERA, Carlos \& SORIA, Jaime. Pregunta de investigación y estrategia PICOT. Medicina. 19. 66-69. 10.23878/medicina.v19i1.647, 2015.

PRESTES, J. et al. Comparison of linear and reverse linear periodization effects on maximal strength and body composition. J of Strength and Cond Res; 23: 266-274, 2016.

PUSPONEGORO, Hardiono D. et al. Gross motor profile and its association with socialization skills in children with autism spectrum disorders. Pediatrics \& Neonatology, v. 57, n. 6, p. 501-507, 2016.

RELVAS, M.P. Neurociências e transtornos de aprendizagem: as múltiplas eficiências para uma educação inclusiva. Sao Paulo. 6 ed. Wak. 2015.

SRINIVASAN, S. M.; BHAT, A. N. Differences in means-end exploration between infants at risk for autism and typically developing infants in the first 15 months of life. Dev. Psychobiol. V. 61 (2): 203-215, 2019.

STEVENSON, J. L.; LINDLEY, C. E.; MURLO, N. Retrospectively assessed early motor and current pragmatic language skills in autistic and neurotypical children. Perceptual and Motor Skills. 124(4):777-794, 2017

TRAVERS, B. G.; BIGLER, E. D.; DUFFIELD, T. C.; PRIGGE, M. D. B.; FROEHLICH, A. L.; LANGE, N.; ALEXANDER, A. L.; LAINHART, J. E. Longitudinal development of manual motor ability in autism spectrum disorder from childhood to mid-adulthood relates to adaptive daily living skills. Developmental Science. V.20, issue 4: 1-15, 2017.

WANG, Z. Individuals with autism spectrum disorder show abnormalities during initial and subsequent phases of precision gripping. J Neurophysiol. Apr 1;113(7):1989-2001.2015.

WATT, N., WETHERBY, A., BARBER, A., \& MORGAN, L. Repetitive and stereotyped behaviors in children with autism spectrum disorders in the second year of life. Journal of Autism and Developmental Disorders, 38(8), 1518-1533, 2008.

WHITEMORE, Robin; KNAFL, Kathleen. The integrative review: updated methodology. Journal of Advanced Nursing, v.52, n.5, p. 546-553, 2005.

WILSON, R.B.; Mc CRACKEN, J.T.; RINEHART, N.J.; JESTE, S.S. What's missing in autism spectrum disorder motor assessments? J Neurodevelop Disord 10, 33. 2018.

ZDROWIA, L. O. International Classification of Functioning, Disability and Health: Children and Youth Version: ICF- CY. Geneva, Switzerland: World Health Organization; 2007. 